\title{
VECTORCARDIOGRAMS OF NORMAL AND PREMATURE BEATS IN DIFFERENT LEAD SYSTEMS
}

\author{
BY \\ W. DEN BOER, H. C. BURGER, AND J. B. VAN MILAAN
}

From the Department of Medical Physics and the Physical Laboratory of the University, Utrecht, Netherlands

Received April 5, 1954

From previous experiments (Burger, van Milaan, and Den Boer, 1952) it has appeared that the correspondence of vectorcardiograms registered with different lead systems is not always satisfactory. The question may be raised as to the cause of this. A bad correspondence may be ascribed to: (A) an abnormal shape or electrical conduction of the thorax; (B) a very large heart; or (C) other causes.

In some cases, in which the correspondence was conspicuously bad, fluoroscopy excluded an abnormal position or size of the heart as the cause. In deciding between these three possibilities the comparison of normal beats and ventricular premature beats can give us some further information. By means of premature beats, nature enables us to make observations in cases in which the excitation proceeds through the heart in quite different ways, while the surroundings of the heart remain entirely unaffected.

Method. Vectorcardiograms were registered in 16 subjects among whom there were some with a pathological vectorcardiogram. In most of them only one type of ventricular extrasystole was observed. When more than one type was present, only the frequently occurring type was used. In each subject vectorcardiograms were registered with the lead systems $B_{1}, R_{2}$, and $W_{4}$ (Burger, van Milaan, and den Boer, 1952).

In system $B_{1}$ the electrodes are connected to the left arm, right arm, and left leg, while a fourth electrode is placed on the sternum at the level of the axilla. In $\mathbf{R}_{2}$ one electrode is connected to the left leg and the other three are at the level of the axilla; one of them is placed centrally on the vertebral column, and the others symmetrically on the thorax at a distance of 50 per cent of the thorax breadth. In both systems the components of the heart vector are linear combinations of the leads with coefficients that have been determined with the aid of a phantom. In system $\mathrm{W}_{4}$, proposed by Wilson et al. (1947), three electrodes are connected to the arms and the left leg, while the fourth is placed on the back at the level of the seventh thoracic vertebra and $2 \mathrm{~cm}$. to the left of the vertebral column. The relation of the heart vector and leads is presented by the authors in geometrical terms. Instead of the equilateral triangle according to Einthoven they use an equilateral tetrahedron of which the frontal plane is the Einthoven triangle, placed vertically. By means of elementary geometry the relation of heart vector and leads can be expressed by an algebraic equation.

\section{RESULTS}

In the ideal case using these systems, the respective vectorcardiograms would be identical. This would hold true for normal as well as for ventricular premature beats. Actually, however, they are not identical. The degree of similarity is expressed as a figure, just as in our previous article; the figure 10 indicates perfect similarity.

The horizontal and frontal projections of the loop have been compared separately, and the 
two figures thus obtained have been averaged. In Fig. 1 (A) and (B) two instances are given. In the first case (A) the similarity in $B_{1}$ and $R_{2}$ of the normal is better than that of the premature beats, while in (B) the reverse is the case. In Fig. 2 the figures expressing similarity of the normal beats

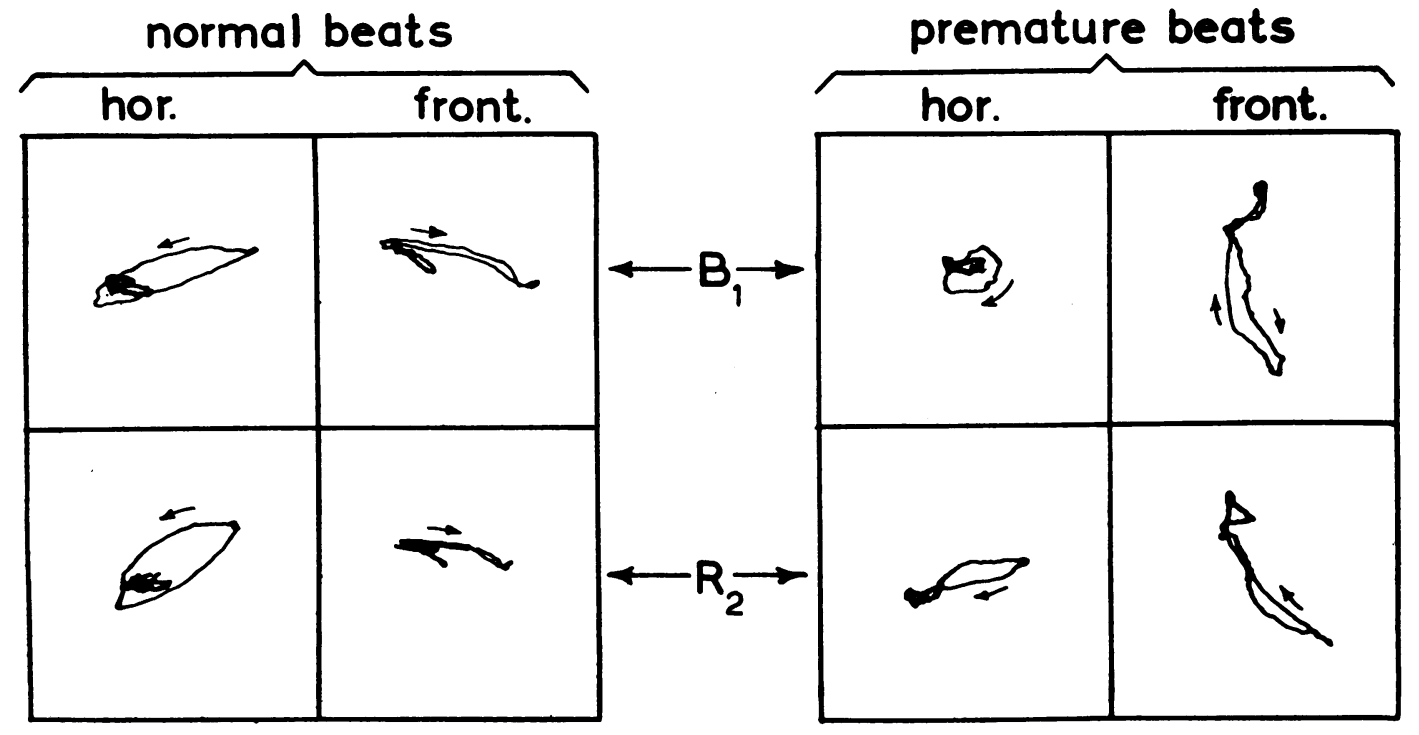

FIG. 1A.-Comparison of the respective normal and premature beats in the systems $\mathbf{B}_{1}$ and $\mathbf{R}_{2}$. The degree of correspondence of normal beats is greater than that of premature beats.

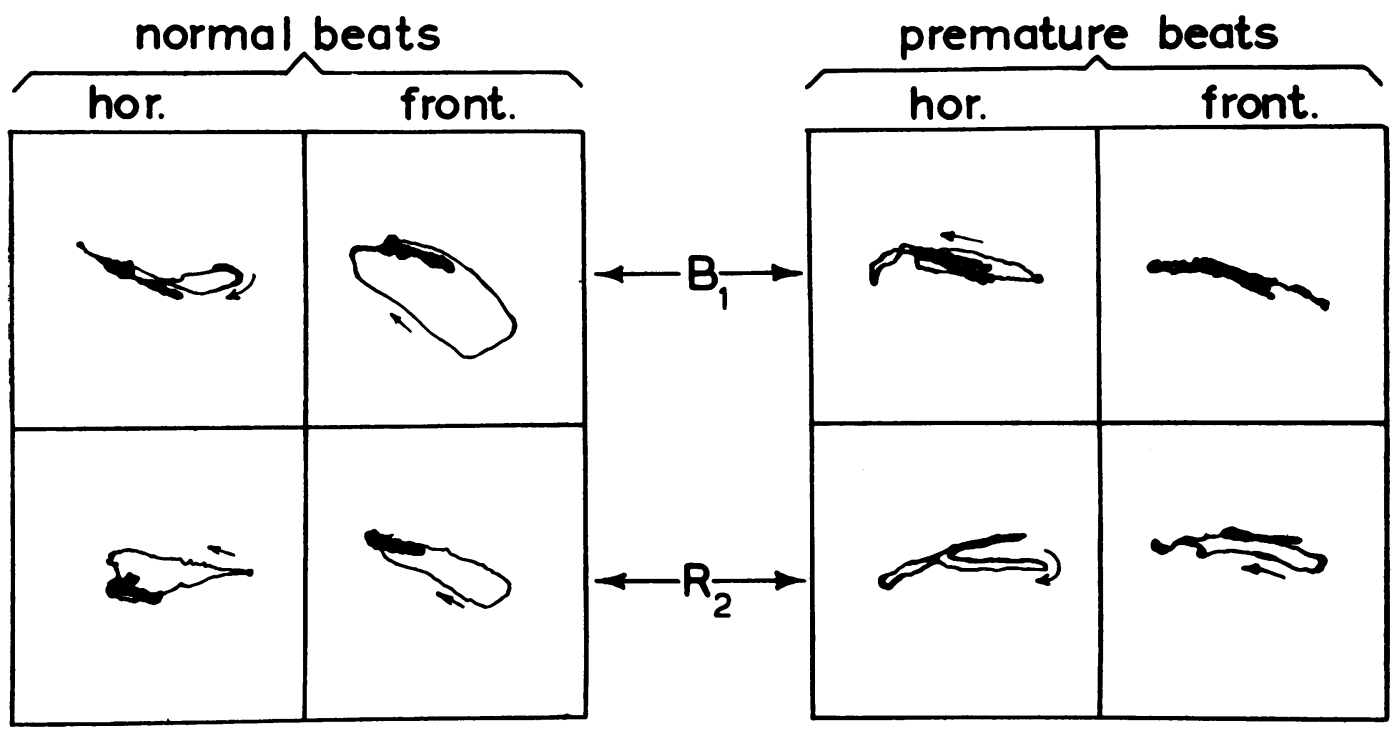

Fig. 1B.-Comparison of the respective normal and premature beats in the systems $\mathbf{B}_{1}$ and $\mathbf{R}_{2}$. The degree of correspondence of premature beats is greater than that of normal ones.

in $B_{1}$ and $R_{2}$ are plotted against those of the premature beats. In order to include also the system of the equilateral tetrahedron $\left(\mathrm{W}_{4}\right)$ in our considerations the averages of the three figures expressing the similarity of $R_{2}$ and $B_{1}, R_{2}$ and $W_{4}$, and of $W_{4}$ and $B_{1}$ are plotted in Fig. 3 .

Conclusion. Fig. 2 and 3 show that there is no correlation between the figures for normal 
beats and extrasystoles. With a clear correlation the dots would form a more or less straight line making an angle of about $45^{\circ}$ with the axes. This would imply that a good correspondence of the normal beats, as a rule would be accompanied by a good correspondence of the premature beats, as in Cases 10 and 15 in Fig. 2. But the correlation is so slight, in fact, that there are cases in which a good correspondence of normal beats goes with a bad correspondence of the premature ones $(8,12,3)$, while the reverse may also occur $(7)$.

\section{Discussion}

The lack of an appreciable correlation indicates that the cause of non-identical vectorcardiograms in the different leads cannot be attributed to circumstances outside the heart, for these circumstances do not change at all when an extrasystole occurs. Nor can the size of the heart be of paramount importance. In the case of a large heart the excitation follows a course that is no longer insignificantly small with respect to the dimensions of the thorax. This will yield a lack of

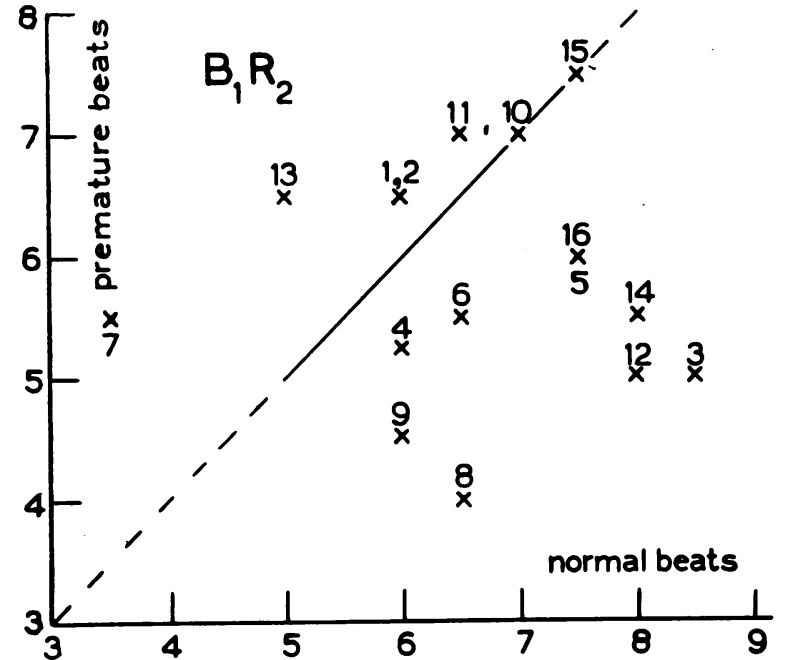

FIG. 2.-Similarity of the respective normal and premature beats in the systems $B_{1}$ and $R_{2}$.

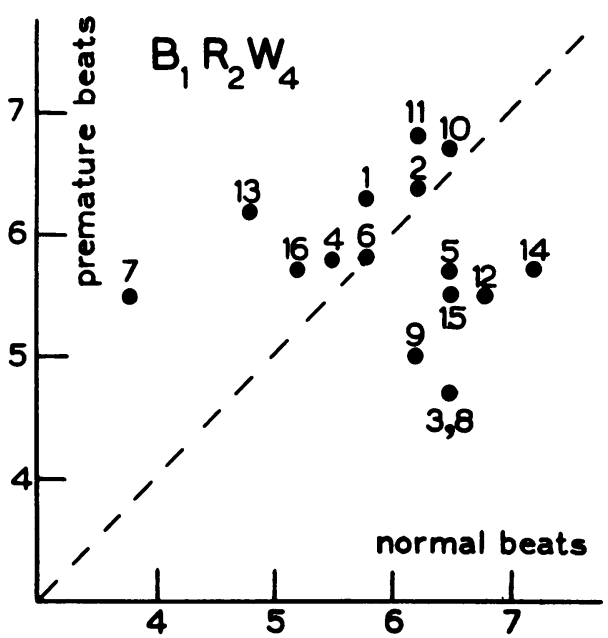

FIG. 3.- Similarity of the respective normal and premature beats in the systems $B_{1} \mathbf{R}_{2}, \mathbf{R}_{2}$ $\mathrm{W}_{4}$, and $\mathrm{W}_{4} \mathrm{~B}_{1}$.

correspondence in the different systems, not only of normal beats but also of extrasystoles. For this reason it cannot be expected that the discrepancy between the similarity of respective normal and premature beats can be explained by cardiac enlargement, which was excluded by fluoroscopy in the above-mentioned cases. So another explanation has to be looked for. It might be found in the occurrence of insulating local adipose tissue on the surface or in the neighbourhood of the myocardium. It is possible that this fatty tissue, because of its uneven distribution round the heart, affects the current field in the thorax differently in the case of a premature and a normal beat, as in each of these two cases the excitation proceeds differently through the heart.

In Fig. 2 it is remarkable that the similarity in $B_{1}$ and $R_{2}$ of normal beats is greater than that of premature ones. This might be accounted for by bearing in mind that in the phantom experiments an average position for the heart was assumed; with the eccentric origin of extrasystoles this assumption is not so valid. So it is probable that local factors can have a noticeable influence on the vectorcardiogram. It should be remembered, however, that this then also holds for the electrocardiogram. 


\section{SUMMARY}

In 16 subjects the degree of correspondence of the different vectorcardiographic lead systems $\left(B_{1}, R_{2}, W_{4}\right)$ has been investigated in the case of both normal and premature beats. No correlation between the degree of correspondence of normal beats and that of premature beats was found. This makes it probable that the bad correspondence was due to local factors such as the uneven distribution of adipose tissue around the heart.

\section{REFERENCES}

Burger, H. C., van Milaan, J. B., and den Boer, W. (1952). Brit. Heart J., 14, 401. Wilson, F. N., Johnston, F. D., and Kossman, C. E. (1947). Amer. Heart J., 33, 594. 